\title{
Steerable spatial phase shifting applied to single-image closed-fringe interferograms
}

\author{
Juan Antonio Quiroga, ${ }^{1, \star}$ Manuel Servin, ${ }^{2}$ Julio Cesar Estrada, ${ }^{2}$ \\ and Jose Antonio Gomez-Pedrero ${ }^{1}$ \\ ${ }^{1}$ Departamento de Optica, Universidad Complutense de Madrid, Ciudad Universitaria S/N, 28040 Madrid, Spain \\ ${ }^{2}$ Centro de Investigaciones en Optica A.C., Leon, Guanajuato 20036, Mexico \\ *Corresponding author: aq@fis.ucm.es
}

Received 22 December 2008; revised 19 March 2009; accepted 19 March 2009; posted 23 March 2009 (Doc. ID 105624); published 16 April 2009

\begin{abstract}
It is well known that spatial phase shifting interferometry (SPSI) may be used to demodulate twodimensional (2D) spatial-carrier interferograms. In these cases the application of SPSI is straightforward because the modulating phase is a monotonic increasing function of space. However, this is not true when we apply SPSI to demodulate a single-image interferogram containing closed fringes. This is because using these algorithms, one would obtain a wrongly demodulated monotonic phase all over the 2D space. We present a technique to overcome this drawback and to allow any SPSI algorithm to be used as a single-image fringe pattern demodulator containing closed fringes. We make use of the $2 \mathrm{D}$ spatial orientation direction of the fringes to steer (orient) the one-dimensional SPSI algorithm in order to correctly demodulate the nonmonotonic 2D phase all over the interferogram. (C) 2009 Optical Society of America OCIS codes: $\quad 050.5080,100.5070,120.5050,120.3180$.
\end{abstract}

\section{Introduction}

In optical metrology, the use of single-image interferogram techniques is of great interest in applications where the use of temporal methods is difficult because of the fast nature of the measured phenomena. In this case, the Fourier transform method can be applied to each single-image interferogram [1]]. Although the Fourier transform method has many advantages, it has two main drawbacks. First, the technique assumes open-fringe interferograms for which the phase is a monotonically increasing function of space and therefore cannot be applied to the general case of single-image closed-fringe interferograms. Second, the processing of interferograms with nonrectangular areas of interest generates errors at the borders, which in turn must be corrected.

In direct space, the spatial phase shifting interferometry (SPSI) methods are a good alternative to the Fourier transform method. However, as with

0003-6935/09/122401-09\$15.00/0

(C) 2009 Optical Society of America the Fourier method, they cannot be applied to the case of single-image closed-fringe patterns with nonmonotonic two-dimensional (2D) modulating phases. To demodulate single-image closed-fringe interferograms in direct space, we can apply regularization techniques [2-4], although they lack the simplicity and processing speed of spatial phase shifting methods. These regularization techniques were the first single-image closed-fringe demodulation methods; however, they have some disadvantages. These problems are mainly the regularization parameter tuning process, the nonlinear character of the phase demodulation, the intensive computing to minimize the local cost function, and the simultaneous computation of phase and spatial frequencies. In this direction, Marroquin et al. [4] were the first to draw attention to the usefulness of decoupling the direction and the spatial frequency computation for the phase demodulation from a single interferogram. This insight was also implicit in the fringe-following regularized phase tracking based methods [2].

The importance of the direction information was explicitly shown by Larkin et al. [5] when they 
demonstrated how the interferogram direction angle [6] can be applied to the Fourier analysis of singleimage closed-fringe interferograms. This technique computes the phase of a single pattern by first applying a frequency filter (the vortex) and then correcting the obtained phase using the direction angle. Also, this method can be used with carrier fringe (openfringe) interferograms in which only the orientation angle is necessary [7]. Onodera et al. [8] presented a 2D Hilbert transform based on direct space discretization of the vortex filter where, again, the direction is the key to process $2 \mathrm{D}$ closed-fringe interferograms.

In this work, we demonstrate how the orientationdirection information can be used to generalize any one-dimensional (1D) SPSI method to the general case of demodulating single-image closed-fringe interferograms with nonmonotonic 2D phase all over the area of interest. The proposed technique shares the simplicity and speed of the spatial SPSI methods, making possible the extension of any SPSI method to the demodulation of closed-fringe single-image interferograms. Working in direct space, it can naturally process irregular areas of interest with minimized border effects. In this way the proposed method is a direct space alternative to the Fourier method for single-image closed-fringe interferograms.

\section{Steerable Spatial Phase Shifting}

A. One-Dimensional Spatial Phase Shifting Interferometry and the Sign Problem

When thinking about the use of SPSI methods, the simplest case is a monochromatic 1D interferometric signal given by

$$
s(x)=b(x)+m(x) \cos \left(\phi_{0}+\omega_{0} x\right),
$$

where $b$ and $m$ are the background and modulation signals, $\omega_{0}$ is a constant spatial frequency, and $\phi_{0}$ is the modulating phase in which we are interested. In this case, the modulating phase is a lineally increasing function of the space, and therefore it can be demodulated using synchronous SPSI methods. All synchronous SPSI techniques can be described by a quadrature filter $g(x)$, tuned at $\omega_{0}$, with a frequency response $G(\omega)=\mathrm{FT}[g(x)]$ that fulfills

$$
G(0)=0, \quad G\left(\omega_{0}\right)=0, \quad G\left(-\omega_{0}\right) \neq 0 .
$$

Due to the Hermitian symmetry of the Fourier transform of a real signal and the fact that the signal is monotonic, the negative (or positive) frequencies are superfluous. Equation (2) represents the basic idea behind any quadrature filter; by eliminating the negative frequencies of the signal spectrum, it is possible to obtain a complex representation from which certain attributes of the signal become more accessible, for example, the phase, to facilitate the derivation of demodulation techniques. If we apply the generic filter described by Eq. (2) to the monochromatic fringe pattern of Eq. (1), we generate a complex phasor given by

$$
A(x)=s(x) * g(x)=m(x) G\left(-\omega_{0}\right) e^{-i\left(\phi_{0}+\omega_{0} x\right)},
$$

from which it is possible to compute the modulating phase by an arctangent calculation.

A typical example of a synchronous SPSI method is the three-step synchronous technique [9], which can be described by the complex filter

$$
\begin{aligned}
g(x)= & {[2 \delta(x)-\delta(x-1)-\delta(x+1)]\left(1-\cos \omega_{0}\right) } \\
& +i[\delta(x-1)-\delta(x+1)] \sin \omega_{0},
\end{aligned}
$$

with frequency response

$$
G(\omega)=4 \sin \left(\frac{\omega-\omega_{0}}{2}\right) \sin \omega .
$$

We can go one step forward in signal complexity and consider a more general type of interferogram given by

$$
s(x)=b(x)+m(x) \cos \left[\phi(x)+\omega_{0} x\right],
$$

for which the phase is no longer constant but fulfills $|\mathrm{d} \phi / \mathrm{d} x| \ll\left|\omega_{0}\right|$, that is to say, the total phase is not linear, but monotonically increasing in space, with local spatial frequency $\omega(x)=\mathrm{d}\left[\phi(x)+\omega_{0} x\right] / \mathrm{d} x>0$. In this case, we say that $s(x)$ is a quasi-monochromatic interferogram. To demodulate this signal, we can no longer use synchronous techniques. Instead, we should use a detuning insensitive SPSI method tuned at $\omega_{0}$, with a frequency response satisfying

$$
G(0)=0, \quad G\left(\omega \approx \omega_{0}\right)=0, \quad G\left(\omega \approx-\omega_{0}\right) \neq 0 .
$$

Again, as the phase is monotonic with $\omega>0$, the positive spatial frequencies are superfluous and can be filtered. In this case, the returned complex phasor is

$$
\begin{aligned}
& A(x)=s(x) * g(\omega)=m(x) G(\omega) e^{-i\left(\phi(x)+\omega_{0} x\right)} \\
& \quad \text { for } \omega \approx \omega_{0} .
\end{aligned}
$$

Again, we can demodulate the interferogram phase from the phasor given by Eq. (8) by an arctangent operation. A typical example of a detuning insensitive method is the five-step Hariharan method [10]. This filter is tuned at $\omega_{0}=\pi / 2 \mathrm{rad} / \mathrm{pixel}$ and can be described by the next filter,

$$
\begin{aligned}
g(x)= & {[2 \delta(x)-\delta(x-2)-\delta(x+2)] } \\
& +2 i[\delta(x-1)-\delta(x+1)],
\end{aligned}
$$

with frequency response

$$
G(\omega)=-2 \sin \omega(\sin \omega-1) .
$$

This filter fulfills Eq. (7) around $\omega_{0}=\pi / 2$, from which it has its detuning insensitive behavior. 
Finally, the more general case of a $1 \mathrm{D}$ interferometric signal is

$$
s(x)=b(x)+m(x) \cos [\phi(x)] .
$$

In this case, the instantaneous spatial frequency, $\omega(x)=\mathrm{d} \phi / \mathrm{d} x$, is only limited by the sampling theorem, $0 \leq|\omega| \leq \pi$. Therefore the interferogram phase is no longer monotonic and can behave arbitrarily. To demodulate this signal, we need a filter that suppresses all the positive spatial frequencies as well as the DC term. This would be a filter with a response given by

$$
G(\omega>0)=0, \quad G(\omega \leq 0) \neq 0 .
$$

In this case, the complex phasor returned is

$$
A(x)=m(x) G(\omega) e^{-i \phi(x)} .
$$

A good example for a filter of this type is the asynchronous five-step method [11]. In this case, the interferogram is assumed to be locally monochromatic with discrete samples given by $s_{k}=b+m \cos [\phi+(k-2) \omega], \quad k=1, \ldots, 5, \quad$ and the phase can be obtained as

$$
\tan \phi=\frac{\operatorname{sign}\left(s_{2}-s_{4}\right) \sqrt{4\left(s_{2}-s_{4}\right)^{2}-\left(s_{1}-s_{5}\right)^{2}}}{2 s_{3}-\left(s_{1}+s_{5}\right)} .
$$

This SPSI method is not linear; therefore the frequency response cannot be computed by a Fourier transform. However, for nonlinear methods, the frequency response can be calculated as the amplitude output of a signal given by $t(x)=\cos \omega x$. In the case of the five-step asynchronous SPSI, the frequency response is

$$
G(\omega)=4 \sin ^{2}(\omega), \omega>0 .
$$

From the former discussion, in general any 1D SPSI filter will have the form

$$
g(s)=f(s)+i h_{1}(s),
$$

where $f(s)$ is an even, real high-pass filter that suppresses the background term $f(b+m \cos \omega x)=$ $-m F(\omega) \cos \omega x$ and $h_{1}(s)$ an odd real filter that transforms the cosine in a sine, that is, a general Hilbert operator with $h_{1}(b+m \cos \omega x)=-m H_{1}(\omega) \sin \omega x$. The filter spectrum is

$$
G(\omega)=F(\omega)+i H_{1}(\omega) .
$$

Additionally, as the general SPSI method $g$ of Eq. (16) is a quadrature filter, it is required that $|F(\omega)|=\left|H_{1}(\omega)\right|$ for a given range of spatial frequencies. For example, if $|F(\omega)|=\left|H_{1}(\omega)\right|$ for a single spatial frequency $\omega_{0}$, we speak of synchronous methods, and if $|F(\omega)|=\left|H_{1}(\omega)\right|$ for $0 \leq \omega \leq \pi$, we say that the method is asynchronous. Therefore, given the symmetries of $f$ and $h_{1}$ and the relation between their spectra, $G$ is a real filter that nulls a range of spatial frequencies.

For example, in the case of the asynchronous fivestep method described above,

$$
\begin{aligned}
h_{1}(s) & =\operatorname{sign}\left(s_{2}-s_{4}\right) \sqrt{4\left(s_{2}-s_{4}\right)^{2}-\left(s_{1}-s_{5}\right)^{2}} \\
& =4 m \sin ^{2} \omega \sin \phi, \\
f(s) & =2 s_{3}-\left(s_{1}+s_{5}\right)=4 m \sin ^{2} \omega \cos \phi,
\end{aligned}
$$

with $|F(\omega)|=\left|H_{1}(\omega)\right|=4 m \sin ^{2} \omega, \omega>0$.

Summarizing, all SPSI methods, linear or not, generate a complex phasor from which the phase can be extracted by an arctangent calculation. The range of frequencies for which the phasor are well calculated depends on the type of SPSI method. Moreover, the election depends on the frequency contents of the interferogram.

However, in the general case of a nonmonotonic phase, it is important not to forget an important detail: all the SPSI methods assume that the modulating phase is monotonic, which means that all the spatial frequencies have the same sign. As a result, the recovered phase is also monotonic. In the case of a nonmonotonic phase, all SPSI methods will demodulate a wrong monotonic phase, losing in this way the phase sign.

Analytically, we can obtain this result using the general quadrature transform. This operator can be expressed as a generalized Hilbert filter multiplied by the fringe direction angle. From [12], the general expression for the Hilbert transform in the $N$-dimensional case is

$$
\mathbf{h}_{N}(s)=\frac{\nabla s}{|\nabla \phi|}=-m \sin \phi \frac{\boldsymbol{\omega}}{|\boldsymbol{\omega}|}=-m \sin \phi \cdot \mathbf{n},
$$

where $\boldsymbol{\omega}=\left(\partial \phi / \partial x_{1}, \ldots, \partial \phi / \partial x_{N}\right)$ is the $N$-dimensional spatial frequency vector, and $\mathbf{n}=\boldsymbol{\omega} /|\boldsymbol{\omega}|$ is the fringe direction vector [6]. In the $1 \mathrm{D}$ case the general expression (19) reduces to

$$
\begin{aligned}
h_{1}(s) & =\frac{\mathrm{d} s / \mathrm{d} x}{\mathrm{~d} \phi / \mathrm{d} x}=-m \sin \phi \frac{\omega}{|\omega|} \\
& =-m \sin \phi \cdot \operatorname{sign}(\omega) .
\end{aligned}
$$

From Eq. (19), we see that the general expression for the Hilbert transform is a nonlinear operator. Also, Eq. (19) states that to obtain the quadrature term of a interferogram, it is always necessary to correct the Hilbert transform by the direction vector $\mathbf{n}$,

$$
q_{N}(s)=\mathbf{h}_{N}(s) \cdot \mathbf{n}=-m \sin \phi,
$$

where $q_{N}(s)$ is the $N$-dimensional quadrature signal corresponding to $m \cos \phi$. In the 1D case we have 


$$
q_{1}(s)=h_{1}(s) \cdot \operatorname{sign}(\omega)=-m \sin \phi .
$$

Therefore all Hilbert transform implementations have to deal with the spatial frequency sign. In the frequency domain, the Hilbert transform can be efficiently implemented by an $N$-dimensional Reis transform $[5,12]$ that has a uniform frequency response with $|H(\boldsymbol{\omega})|=1$. However, in the space domain with a limited number of samples, we have only approximations to this behavior.

From this discussion, in the case of a 1D nonmonotonic signal, the complex phasor returned by any SPSI method must be corrected by the spatial frequency sign in order to compute correctly the phase. Therefore from Eq. (20), the complex phasor that we obtain for any SPSI filter is

$$
A(x)=m(x) G(\omega)[\cos \phi+i \operatorname{sign}(\omega) \sin \phi] .
$$

\section{B. Two-Dimensional Steerable Spatial Phase Shifting}

In the former section, we have shown the importance of the spatial frequency sign in the demodulation of a $1 \mathrm{D}$ signal using any SPSI method. In the case of a $2 \mathrm{D}$ closed-fringe interferogram, the role of the spatial frequency sign is assumed by the fringe direction angle. From the general expression of the Hilbert transform given by Eq. (19), the components of the Hilbert transform in the case of a general 2D interferogram given by

$$
s(x, y)=b(x, y)+m(x, y) \cos [\phi(x, y)]
$$

are

$$
\begin{aligned}
h_{2 x}[s(x, y)] & =\frac{\mathrm{d} s / \mathrm{d} x}{|\nabla \phi|}=-m \sin \phi \frac{\omega_{x}}{|\omega|} \\
& =-m \sin \phi \cos \beta, \\
h_{2 y}[s(x, y)] & =\frac{\mathrm{d} s / \mathrm{d} y}{|\nabla \phi|}=-m \sin \phi \frac{\omega_{y}}{|\omega|} \\
& =-m \sin \phi \sin \beta,
\end{aligned}
$$

where $\beta$ is the fringe direction angle, defined as the angle subtended by the fringe direction vector with the $x$ axis [6]. From Eq. (25), if we apply any SPSI filter $g$ along the $x$ coordinate to a $2 \mathrm{D}$ interferogram, we obtain a phasor given by

$$
A_{x}(x, y)=m(x, y) G\left(\omega_{x}\right)[\cos \phi+i \cos \beta \sin \phi] .
$$

Therefore the recovered quadrature term is modulated by $\cos \beta$, and if $\omega_{x} \approx 0(\cos \beta \approx 0)$, then the recovered phase is zero independently of $\phi$. The same problem appears if we apply the same SPSI method in the $y$ direction. In this case the phasor signal is

$$
A_{y}(x, y)=m(x, y) G\left(\omega_{y}\right)[\cos \phi+i \sin \beta \sin \phi],
$$

and the quadrature term is modulated by $\sin \beta$. Therefore, for $\omega_{y} \approx 0(\sin \beta \approx 0)$, the recovered phase is zero independently of $\phi$.

A closed-fringe interferogram has a nonmonotonic phase with positive and negative $2 \mathrm{D}$ spatial frequencies in $x$ and $y$. If we apply a 1D SPSI method, even in the case that we do correct for the direction angle, we have a wrongly demodulated phase.

The solution for this problem is to compute the $2 \mathrm{D}$ quadrature term incorporating the direction information, "steering" in this way the 1D SPSI filter along the fringe direction. Then, if $g(s)=$ $f(s)+i h(s)$ is a $1 D$ SPSI filter, the quadrature term is obtained using Eq. (21) as

$$
\begin{aligned}
q_{2}(s) & =\left[h_{2 x}(s), h_{2 y}(s)\right] \cdot \mathbf{n} \\
& =h_{2 x}(s) \cos \beta+h_{2 y}(s) \sin \beta \\
& =-m\left[G\left(\omega_{x}\right)+G\left(\omega_{y}\right)\right] \sin \phi,
\end{aligned}
$$

where $h_{2 x}$ and $h_{2 y}$ correspond to the same Hilbert filter applied in the $x$ and $y$ directions, respectively. From Eq. (28), the general formula to "steer" any $1 \mathrm{D}$ SPSI method is

$$
\tan \phi=\frac{h_{2 x}(s) \cos \beta+h_{2 y}(s) \sin \beta}{f_{x}(s)+f_{y}(s)},
$$

and the frequency response of the method is $G(\boldsymbol{\omega})=G\left(\omega_{x}\right)+G\left(\omega_{y}\right)$.

For example, if we assume that locally our interferogram is monochromatic, we can always express it as

$$
\begin{aligned}
s(k, l) & =b+m \cos \left[\phi+(k-2) \omega_{x}+(l-2) \omega_{y}\right], \\
k, l & =1, \ldots, 5,
\end{aligned}
$$

and we can apply the five-step asynchronous method [11] along rows

$$
\begin{aligned}
h_{2 x}(s) & =\operatorname{sign}\left(s_{2, l}-s_{4, l}\right) \sqrt{4\left(s_{2, l}-s_{4, l}\right)^{2}-\left(s_{1, l}-s_{5, l}\right)^{2}} \\
& =4 m \sin ^{2} \omega_{x} \cos \beta \sin \phi, \\
f_{x}(s) & =2 s_{3, l}-\left(s_{1, l}+s_{5, l}\right)=4 m \sin ^{2} \omega_{x} \cos \phi,
\end{aligned}
$$

and along columns

$$
\begin{aligned}
h_{2 y}(s) & =\operatorname{sign}\left(s_{k, 2}-s_{k, 4}\right) \sqrt{4\left(s_{k, 2}-s_{k, 4}\right)^{2}-\left(s_{k, 1}-s_{k, 5}\right)^{2}} \\
& =4 m \sin ^{2} \omega_{x} \sin \beta \sin \phi, \\
f_{y}(s) & =2 s_{k, 3}-\left(s_{k, 1}+s_{k, 5}\right)=4 m \sin ^{2} \omega_{y} \cos \phi .
\end{aligned}
$$

Finally, the phase is computed using Eq. (29). For this 2D SPSI method the frequency response is $G(\boldsymbol{\omega})=4 m\left(\sin ^{2} \omega_{x}+\sin ^{2} \omega_{y}\right)$.

Another example is the five-step Hariharan method [10] that was designed for $\omega_{x}, \omega_{y} \approx$ $\pi / 2 \mathrm{rad} / \mathrm{sample}$. In this case, the SPSI filter is given by Eq. (9) along rows 


$$
\begin{aligned}
h_{2 x}(s) & =2\left(s_{2, l}-s_{4, l}\right)=4 m \sin ^{2} \omega_{x} \cos \beta \sin \phi, \\
f_{x}(s) & =2 s_{3, l}-\left(s_{1, l}+s_{5, l}\right)=4 m \sin ^{2} \omega_{x} \cos \phi,
\end{aligned}
$$

and along columns

$$
\begin{aligned}
h_{2 y}(s) & =2\left(s_{k, 2}-s_{k, 4}\right)=4 m \sin ^{2} \omega_{y} \sin \beta \sin \phi, \\
f_{y}(s) & =2 s_{k, 3}-\left(s_{k, 1}+s_{k, 5}\right)=4 m \sin ^{2} \omega_{y} \cos \phi .
\end{aligned}
$$

Again, the phase is computed using Eq. (29). For this method, outside the range $\omega_{x}, \omega_{y} \approx \pi / 2$ the recovered phase presents periodic detuning errors, and the frequency response is $G(\boldsymbol{\omega})=4 m\left(\sin ^{2} \omega_{x}+\sin ^{2} \omega_{y}\right)$, $\omega_{x}, \omega_{y} \approx \pi / 2$. It is not surprising that the frequency response coincides with that of the five-step nonlinear asynchronous method of Eqs. (31) and (32). In fact, for $\omega \approx \pi / 2\left(s_{1}-s_{5} \approx 0\right)$, the asynchronous method becomes the linear Hariharan technique.

Although we have centered our discussion on the use of 1D SPSI methods, the presented technique can be generalized to any 1D phase demodulation technique as, for example, the $1 \mathrm{D}$ wavelet transform [13], the windowed Fourier transform [14], or the 1D phase lock loop [15].

Finally, we must mention the effect of using the orientation angle instead of the fringe direction for closed-fringe interferograms. The orientation angle can be computed directly from the irradiance [7]; however, in the case of closed fringes, it cannot describe the complete set of fringe directions. This is because the orientation is defined modulo $\pi$ (from the local irradiance, we do not know if a fringe is directed "left" or "right"); then if $\theta$ is the orientation angle,

$$
\theta=\beta \pm K \pi,
$$

where $K$ is an integer such that $0 \leq \theta \leq \pi$. From Eq. (35),

$$
\cos \theta=(-1)^{K} \cos \beta, \quad \sin \theta=(-1)^{K} \sin \beta .
$$

For open-fringe interferograms, $K=0$, and the use of orientation and direction is equivalent; however, in the case of closed-fringe patterns $K(x, y)=\{0,1\}$, if we apply the steering Eq. (29) with the orientation instead of the direction, we get

$$
\tan \phi_{\theta}=(-1)^{K(x, y)} \tan \phi,
$$

where $\tan \phi_{\theta}$ is the $2 \mathrm{D}$ phase recovered by using the orientation. Therefore from Eq. (37) for a closedfringe interferogram when the direction is bigger than $\pi$ or less than 0 , a sign change appears in the recovered phase. From the point of view of the spatial frequencies, to have access only to the orientation is equivalent to ignoring the sign of the $\omega_{y}$ component of the spatial frequency. As a result, the returned phase will always be monotonic in the $y$ direction with $\omega_{y}>0$ all over $2 \mathrm{D}$ space.

\section{Experimental Results}

In the examples presented in this section, the orientation angle is calculated using the method described in [7], and the direction angle is computed using the technique of Villa et al. [6].

The first example is a computer generated circular fringe pattern that helps to clarify the concepts discussed in the previous sections. We use a $256 \times 256$ interferogram given by

$$
\begin{aligned}
s & =128+128 \cos \left[0.5 \pi\left(x^{2}+y^{2}\right) / 256\right], \\
x, y & =-128, \ldots, 128 .
\end{aligned}
$$

This is a circular chirp signal with a nonmonotonic phase for which the spatial frequency ranges $-0.5 \pi \leq \omega_{x}, \omega_{y} \leq 0.5 \pi$.

Figure 1 shows the test interferogram; in this figure the radial variation of the spatial frequency of the closed fringes is clear. We demodulate the phase of this interferogram by steering the asynchronous SPSI method given by Eqs. (29), (31), and (32). Figure 2 shows the $h_{2 x}(s) \approx \sin \phi$ and $f_{x}(s) \approx \cos \phi$ terms given by Eq. (32). In Fig. 2(a), the $h_{2 x}(s)$ term shows clearly the abrupt jump due to the horizontal spatial frequencies sign as well as the lack of modulation predicted by the $\sin ^{2} \omega_{x}$ factor of Eq. (32). On the other hand, in Fig. 2(b) the $f_{x}(s)$ term shows its even character, but also it is present in the $\sin ^{2} \omega_{x}$ modulation that distorts the circular fringes in the central vertical line for which $\omega_{x} \approx 0$.

Figure 3(a) shows the demodulated phase by means of Eq. (29) but using only the orientation angle. As predicted in Eq. (37), there is a sign jump due to the modulo $\pi$ character of the orientation. Figure 3(b) shows the demodulated phase using the fringe direction angle. In this case, the orientation problem is solved, and the 1D asynchronous SPSI is effectively steered to demodulate the 2D closed-fringe single-image interferogram of Fig. 1. To demonstrate the capability to steer any 1D SPSI,

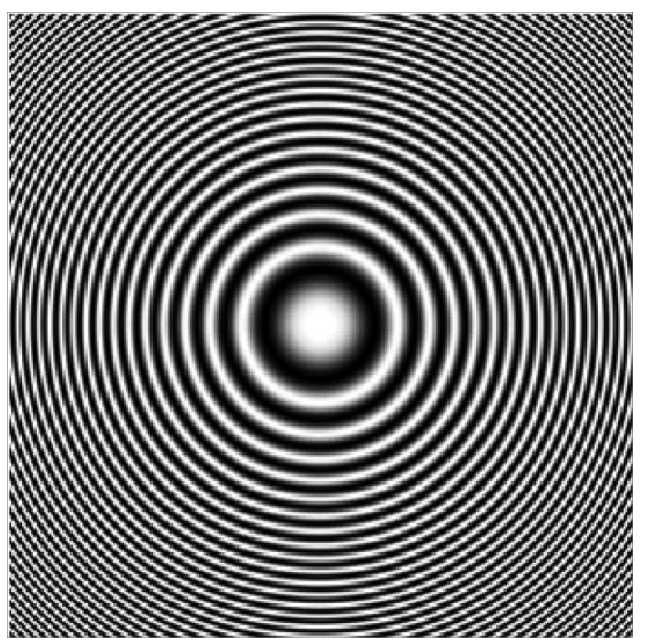

Fig. 1. Computer generated closed-fringe interferogram. 


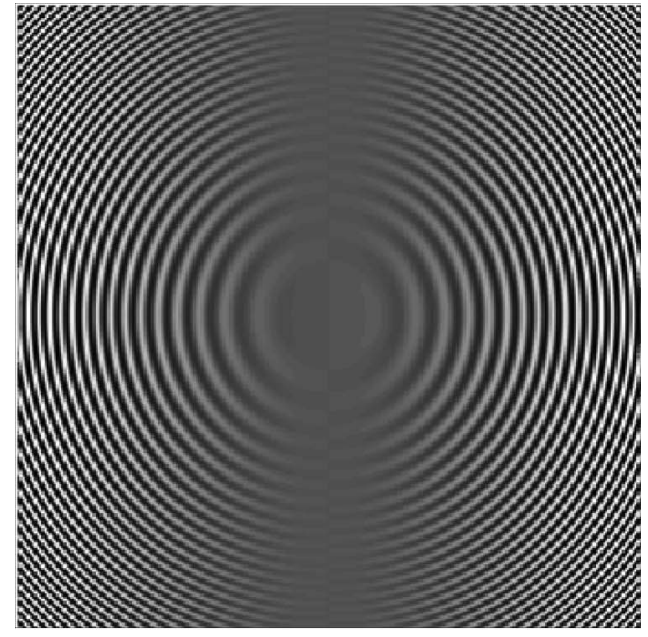

(a)

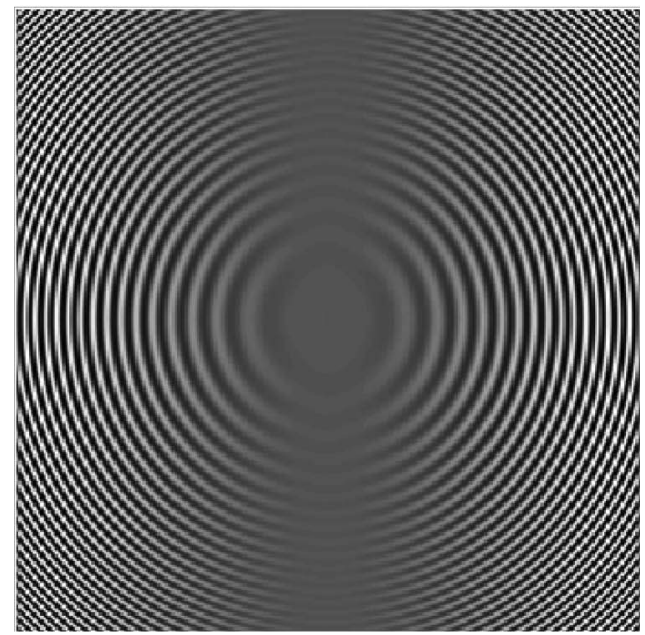

(b)

Fig. 2. Phasor terms obtained from the processing of the interferogram of Fig. 1 by the five-step SPSI asynchronous method applied in the horizontal direction: (a) sine term before the sign correction, (b) cosine term. Note in both images the fringe amplitude toward the central vertical line predicted by the $\sin ^{2} \omega_{x}$ term.

Figs. 4(a) and 4(b) depict the phase demodulated by the steering of the three-step [Eq. (4)] and five-step [Eq. (9)] SPSI methods, respectively. Although there are phase errors, the phase jump locations of the actual phase map are recovered correctly, making possible the use of refinement techniques to recover a better estimation for the demodulated phase [12]. In this example the total processing time including direction computation was $2.7 \mathrm{~s}$.

The next example is a $552 \times 736$ experimental Fizeau interferogram. Figure 5(a) shows the manually masked interferogram, where it is possible to observe two saddle points and a central closed fringe. Figure 5(b) shows the demodulated phase using the asynchronous SPSI method of Eqs. (29), (31), and (32). As can be seen, the steering has been

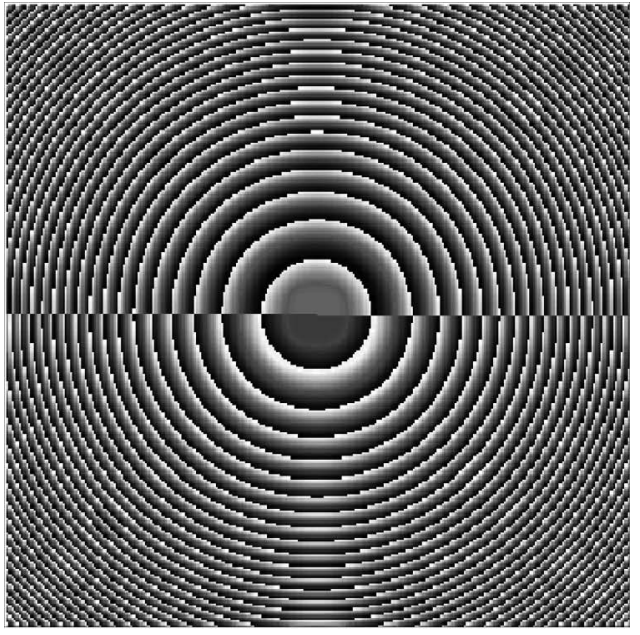

(a)

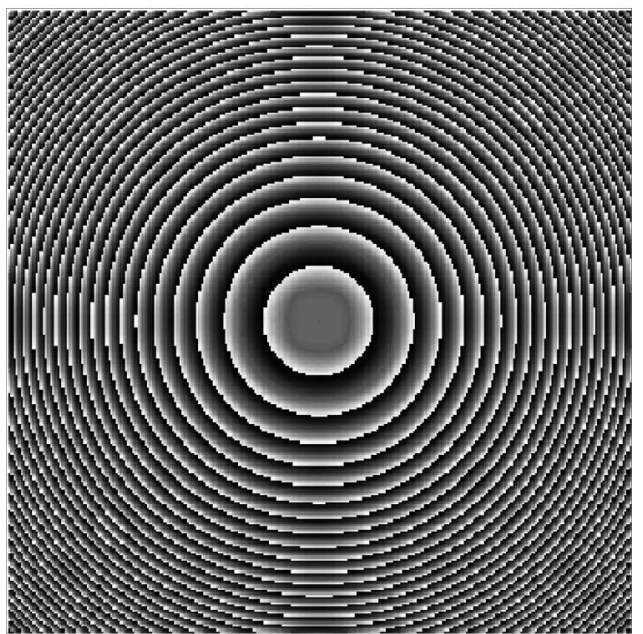

(b)

Fig. 3. Demodulated phase from the interferogram shown in Fig. 1 using the steered five-step SPSI asynchronous technique: (a) using the orientation, note the incorrect demodulation due to the nonmonotonic phase, and (b) using the direction angle.

correct even in the presence of the saddle points. For this example the total computing time was $15.7 \mathrm{~s}$.

The next example is a $240 \times 256$ shadow moiré topography, closed-fringe interferogram. Figure $\underline{6(a)}$ shows the shadow moiré image where one can observe the typical lack of contrast of this technique for the high order fringes and also a vertical scratch due to a grid flaw. In this case, the drawing of a manual mask will be difficult due to the progressive lack of fringe modulation. Figure 6(b) shows the demodulated phase using the steered asynchronous SPSI method of Eqs. (29), (31), and (32). As can be seen, low modulation areas as well as the scratch zone have been processed without affecting their neighborhood. Figure 6(c) shows the amplitude of the complex phasor from which we calculated the phase of Fig. 6(b). This amplitude reflects the quality of the obtained phase. To show this, Fig. 6(d) depicts the phase unwrapping of Fig. 6(b) by using a simple 


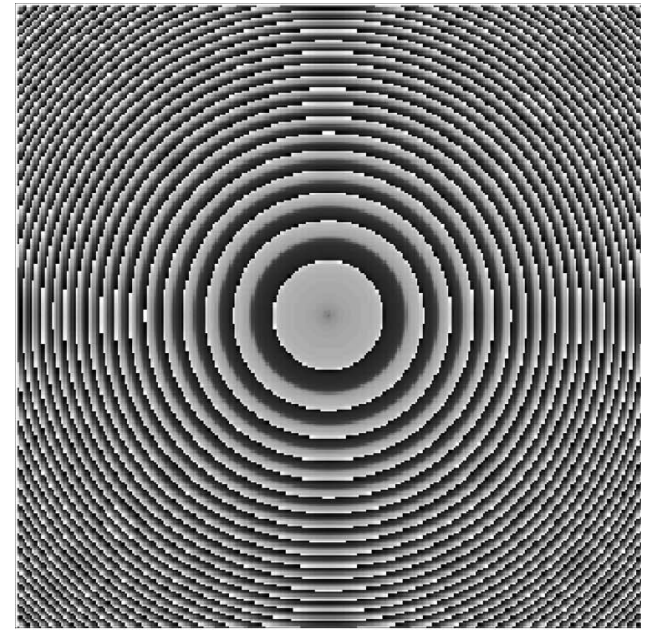

(a)

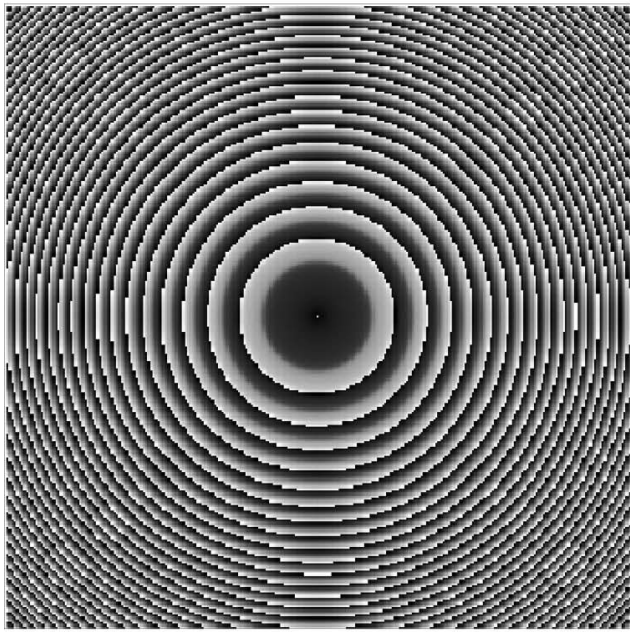

(b)

Fig. 4. Demodulated phase from the interferogram of Fig. 1 using two steered SPSI methods: (a) the five-step Hariharan and (b) the standard three-step SPSI method.

and fast, quality-map guided method [16]. As can be seen, the results are very good, and the steered spatial phase-stepping method makes possible the automatic processing of a closed-fringe interferogram, where the direct application of a SPSI method is not possible and the manual design of a processing mask is not an obvious task. In this example the total processing time was $2.5 \mathrm{~s}$.

Finally we demonstrate the suitability of the proposed technique for noisy fringe patterns. As explained in Section 3, the method is factorized in two parts: the orientation-direction calculation and the 1D SPSI demodulation method. The direction computation method has demonstrated a good behavior with respect to noise [6,7]; in fact, the direction information is low frequency by definition and can be separated very effectively from the noise. Therefore, if we speak about noise, the proposed technique is as good as the 1D SPSI method used. Figure 7(a) depicts a $281 \times 251$ ESPI out-of-plane deformation interfer-

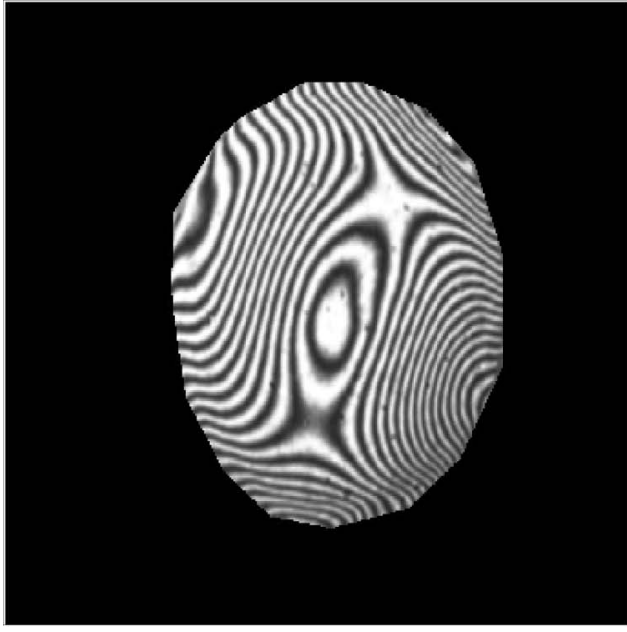

(a)

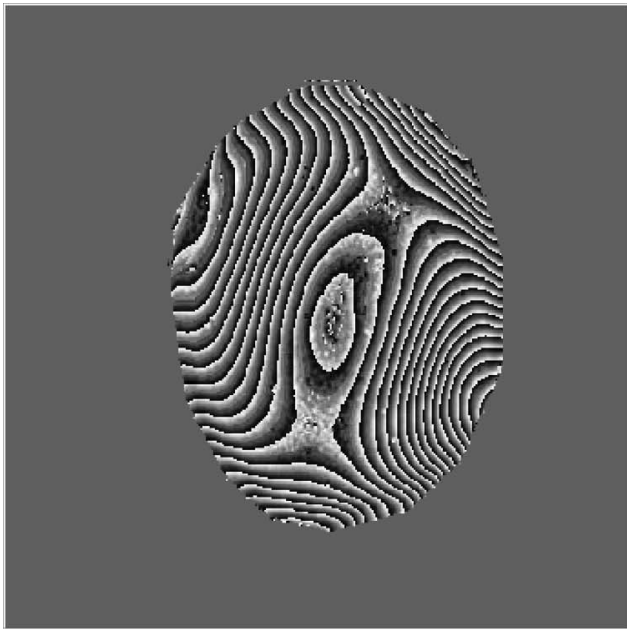

(b)

Fig. 5. (a) Experimental closed-fringe Fizeau interferogram and (b) demodulated phase using the steered five-step asynchronous SPSI method.

ogram. In this case we use the adaptive asynchronous five-sample SPSI method [17]. Figure 7(b) shows the demodulated phase from the fringe pattern of Fig. 1. Figure 7(c) shows the $3 \times 3$ averaging sine-cosine low-pass filtered phase map [16]. Finally, for comparison, Figure 7(d) depicts the E.SPI phase map computed using four temporal phase stepped images. From Fig. 7(c) it is worth noting the sign inversion in the left-side lower bump with respect to the temporal phase stepped image. This is due to the even character of the cosine function that, in the case of nonconnected closed fringes, does not permit the distinction between the situations in which all the bumps are concave, convex, or any concave-convex combination, because the cosine is the same for all solutions. Therefore this sign inversion is not an error but a feature of any single-image closed-fringe interferogram demodulation technique. To demonstrate the correctness of the demodulated phase, Fig. 7(e) shows the cosine of the phase 


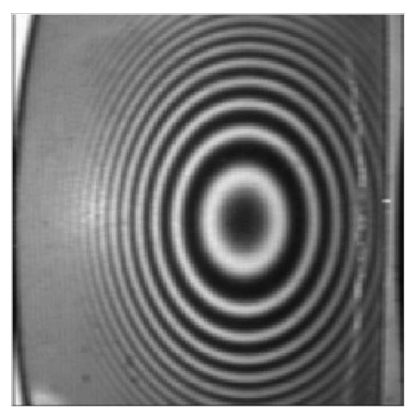

(a)

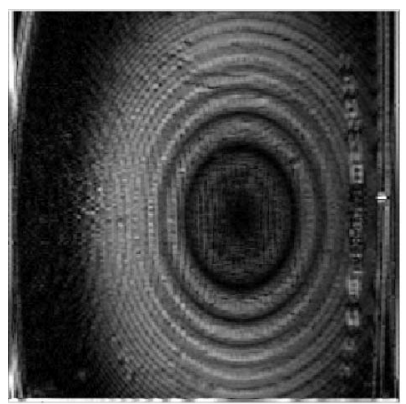

(c)

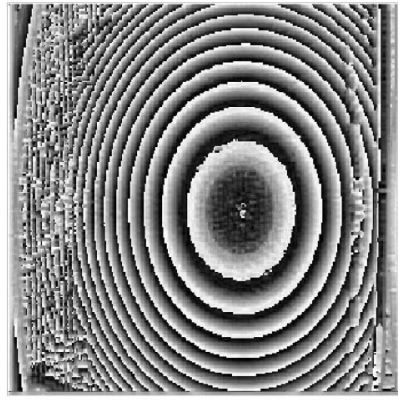

(b)

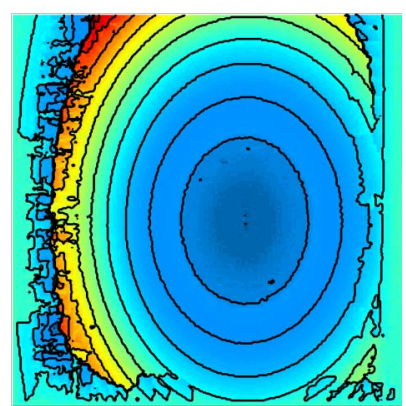

(d)
Fig. 6. (Color online) (a) Experimental shadow moiré topography interferogram, (b) demodulated phase using the steered five-step asynchronous SPSI method, (c) phasor amplitude used as quality map for unwrapping the phase depicted in Fig. 6(b), and (d) unwrapped phase using a quality-guided phase unwrapping method.

depicted in Fig. 7(c). As can be seen, the results obtained by the proposed technique are very satisfactory compared with the temporal technique. In this case the total processing time was $4 \mathrm{~s}$.

From the experimental results presented, we can see that there is a lack of accuracy in the recovered phase at the fringe centers, saddle points, and constant phase regions. The reason is that, at these points, the spatial frequencies are null in both directions. In this case the orientation direction is not defined, and the intensity gradient is null. Under these conditions the computation of the quadrature term $(b \sin \phi)$ from the intensity signal $(b+m \cos \phi)$ is not possible. Mathematically the quadrature transform [Eq. (21)] is not defined if both spatial frequencies are null. Qualitatively, if the local spatial frequency of the interferogram is null, there is no way to separate the background term from the cosinoidal term using a single-image interferogram. Therefore any demodulation technique will have unreliable results at saddle points, fringe centers, and constant phase regions. This problem can only be solved using a temporal carrier (or that is the same using more than one image). For single-image interferograms, this subject can be alleviated incorporating interpolation-smoothing operations that permit the processing of these regions if the low phase gradient region is not big and it is surrounded by reliable fringe data.

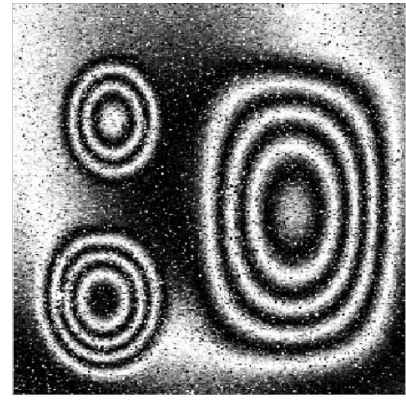

(a)

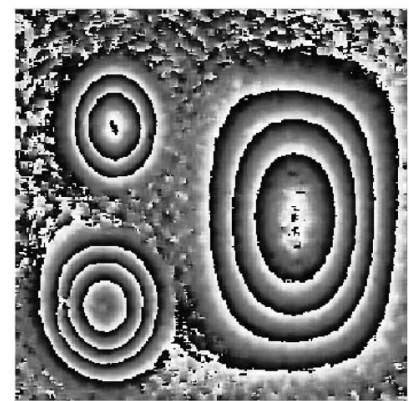

(c)

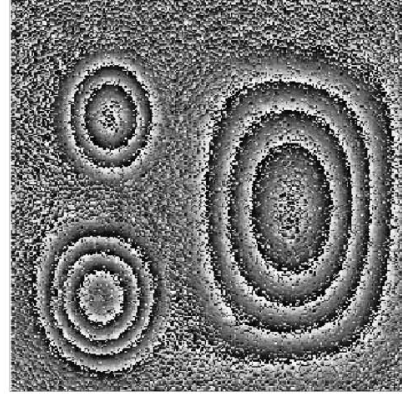

(b)

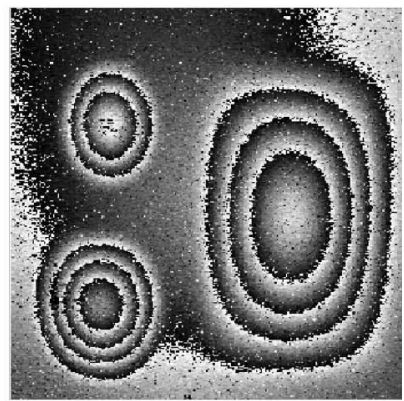

(d)

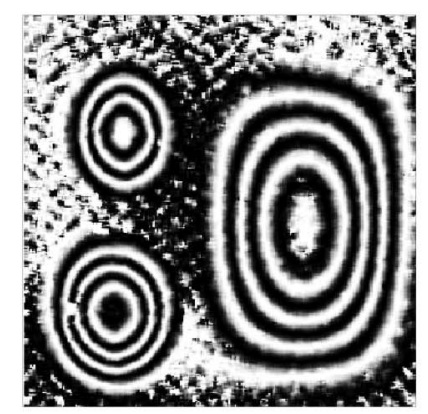

(e)

Fig. 7. (a) Experimental out-of-plane ESPI interferogram, (b) demodulated phase using a noise-rejecting SPSI method [17], (c) $3 \times 3$ averaging sine-cosine low-pass filtered phase map, (d) phase map obtained using four temporal phase-shifted images, (e) cosine of the phase depicted in Fig. 7(c); the comparison with Fig. $\underline{7(a)}$ shows the correctness of the demodulated phase.

\section{Conclusions}

We have presented a method to generalize any $1 \mathrm{D}$ SPSI method to the $2 \mathrm{D}$ case. We have shown that in the general case of single-image closed-fringe interferograms, the nonmonotonic behavior of the phase will make any 1D SPSI method yield an incorrect demodulated phase. To solve this problem, we have shown how the direction information can be used to steer any 1D SPSI filters in order to process single-image closed-fringe interferograms. This makes possible the generalization to the general $2 \mathrm{D}$ case of all the results obtained in SPSI, for example, harmonic rejection and detuning properties. The result can be applied to any $1 \mathrm{D}$ demodulation technique as wavelet or phase lock loop techniques.

Finally, the proposed technique is simple to implement. It has two components, the SPSI technique 
and the orientation-direction computation. The 1D SPSI techniques used can be as simple as the linear methods discussed in this work or as elaborate as a $1 D$ spatial wavelet transform. The orientation calculation is a straightforward arctangent operation using the intensity gradient [7], and the direction computation method is based on a path-following technique with a linear local computation [6]. Therefore, unless a complicated demodulator is used, the 2D generalization of any 1D SPSI method can be done using exclusively linear operations and pathfollowing techniques.

We thank the Ministerio de Ciencia y Tecnología of Spain for the financial support of this work given by project DPI2005-03891. Also M. Servin and J. C. Estrada acknowledge the financial support of the Mexican Consejo Nacional de Ciencia y Tecnologia (CONACYT).

\section{References}

1. M. Takeda and K. Mutoh, "Fourier transform profilometry for the automatic measurement of 3-D object shapes," Appl. Opt. 22, 3977-3982 (1983).

2. M. Servin, J. L. Marroquin, and F. Cuevas, "Fringe-follower regularized phase tracker for demodulation of closedfringe interferograms," J. Opt. Soc. Am. A 18, 689-695 (2001).

3. J. L. Marroquin, M. Rivera, S. Botello, R. Rodriguez-Vera, and M. Servin, "Regularization methods for processing fringepattern images," Appl. Opt. 38, 788-794 (1999).

4. J. L. Marroquin, R. Rodriguez-Vera, and M. Servin, "Local phase from local orientation by solution of a sequence of linear systems," J. Opt. Soc. Am. A 15, 1536-44 (1998).

5. K. Larkin, D. J. Bone, and M. A. Oldfield, "Natural demodulation of two-dimensional fringe patterns. I. General background of the spiral phase quadrature transform," J. Opt. Soc. Am. A 18, 1862-1870 (2001).
6. J. Villa, I. De la Rosa, G. Miramontes, and J. A. Quiroga, "Phase recovery from a single fringe pattern using an orientational vector-field-regularized estimator," J. Opt. Soc. Am. A 22, 2766-2773 (2005).

7. X. Yang, Q. Yu, and S. Fu, "An algorithm for estimating both fringe orientation and fringe density," Opt. Commun. 274, 286-292 (2007).

8. R. Onodera, Y. Yamamoto, and Y. Ishii, "Signal processing of interferogram using a two-dimensional discrete Hilbert transform," in Proceedings of Fringe 2005, Fifth International Workshop on Automatic Processing of Fringe Patterns, W. Osten, ed. (European Space Agency, 2005), pp. 82-89.

9. C. J. Morgan, "Least-squares estimation in phase-measurement interferometry," Opt. Lett. 7, 368-370 (1982).

10. P. Hariharan, B. F. Oreb, and T. Eiju, "Digital phase-shifting interferometry: a simple error-compensating phase calculation algorithm," Appl. Opt. 26, 2504-2506 (1987).

11. J. A. Gomez Pedrero, J. A. Quiroga, and M. Servín, "Asynchronous phase demodulation algorithm for temporal evaluation of fringe patterns with spatial carrier," J. Mod. Opt. 51, 97109 (2004).

12. M. Servin, J. A. Quiroga, and J. L. Marroquín, "A general $n$ dimensional quadrature transform and its applications to interferogram demodulation," J. Opt. Soc. Am. A 20, 925934 (2003).

13. J. Zhong and J. Weng "Spatial carrier-fringe pattern analysis by means of wavelet transform: wavelet transform profilometry," Appl. Opt. 43, 4993-4998 (2004).

14. Q. Kemao, "Two-dimensional windowed Fourier transform for fringe pattern analysis: principles, applications and implementations," Opt. Lasers Eng. 45, 304-317 (2007).

15. M. Servin, R. Rodriguez-Vera, and D. Malacara, "Noisy fringe pattern demodulation by an iterative phase locked loop," Opt. Lasers Eng. 23, 355-365 (1995).

16. B. Strobel, "Processing of interferometric phase maps as complex-valued phasor images," Appl. Opt. 35, 2192-2198 (1996).

17. J. A. Gómez-Pedrero, J. A. Quiroga, and M. Servin, "Adaptive asynchronous algorithm for fringe pattern demodulation," Appl. Opt. 47, 3954-3961 (2008). 